\title{
Reflexiones metodológicas para el análisis de la gestión e indicadores de calidad con personas administradoras educativas
}

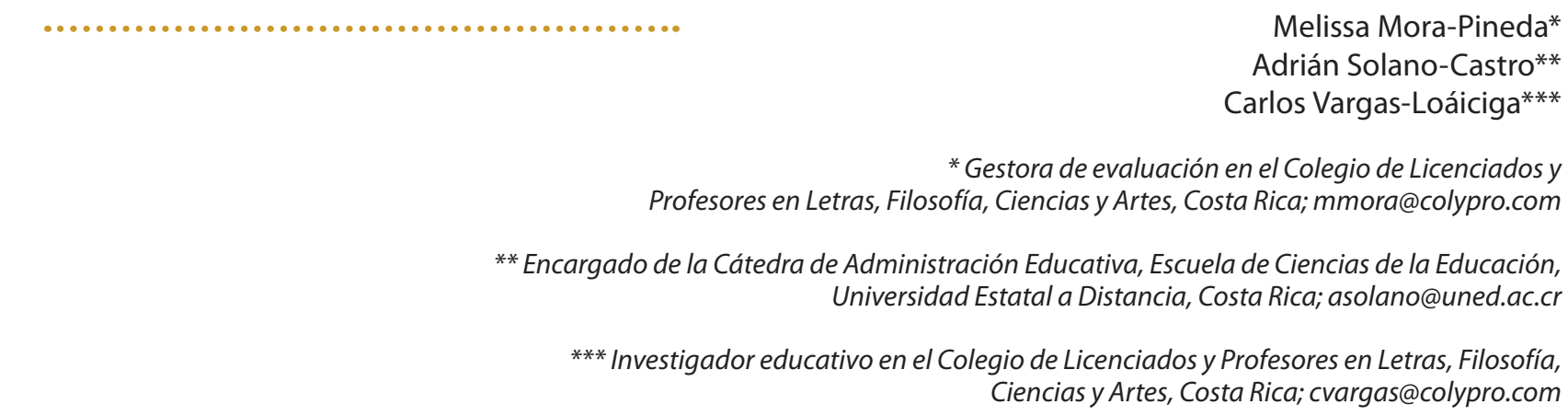

Recibido: 31 de julio del $2019 \quad$ Corregido: 17 de octubre del $2019 \quad$ Aceptado: 28 de octubre del 2019

\begin{abstract}
Resumen
El abordaje metodológico es fundamental en cualquier proceso de investigación, ya que el desarrollo de los procesos de recolección de información permite la construcción sistemática adecuada de resultados que responden los objetivos. Este artículo se centra en la herramienta metodológica del grupo focal, la cual ha demostrado su utilidad como técnica de investigación en diversos contextos y para múltiples disciplinas. A pesar de esto, la literatura sobre su desarrollo en el campo de la Administración Educativa ha sido escasa. Por tanto, se reflexiona acerca de la propuesta teórico-metodológica y se detallan los procesos de organización y planificación del grupo focal. Además, se hace una reflexión a partir de un primer taller, planteado dentro de la investigación que realizan, en conjunto, el personal de la carrera Administración Educativa de la Universidad Estatal a Distancia (UNED) y el Colegio de Licenciados y Profesores en Letras, Filosofía, Ciencias y Artes (Colypro), para el análisis de la gestión e indicadores de calidad con personas administradoras educativas.
\end{abstract}

Palabras claves: Calidad de la educación; investigación-acción; indicadores de calidad; gestión de la educación; grupo focal.

\section{Abstract \\ Methodological reflections for management analysis and quality indicators with educational administrators}

The methodological approach is fundamental in any research process, since the development of the information gathering processes allows the adequate systematic construction of results that meet the objectives. In this article, it centralizes on the methodological tool of the focus group, which has demonstrated it is usefulness as a research technique in differents contexts and for multiple disciplines, however, the literature on its methodological development in the field of Educational Administration has been limited. Therefore, we seek to reflect on the theoretical-methodological proposal where the processes of organization and planning of the focus group are detailed and makes a reflection from the first workshop executed. That was raised within the research carried out by the staff of the Educational Administration career at Universidad Estatal a Distancia (UNED) and Colegio 
de Licenciados y Profesores en Letras, Filosofía, Ciencias y Artes (Colypro) for the analysis of management and quality indicators with educational administrators

Key words: Education quality; action research; quality indicators; education management; focus group.

\section{INTRODUCCIÓN}

Costa Rica tiene en la educación el mecanismo más efectivo de movilización social, de ahí que organismos nacionales e internacionales han elaborado diversos informes para comprender y profundizar en los desafíos educativos más relevantes para la mejora en la calidad educativa. Tal y como lo manifiestan Ramírez y Quesada (2019):

En los últimos años, entidades nacionales como el Programa Estado de la Nación a través de sus informes del Estado de la Educación, así como de las agencias internacionales la Organización para la Cooperación y el Desarrollo Económicos (OCDE) han realizado esfuerzos por investigar y analizar los desafíos educativos y determinar aquellos factores clave que permitan superarlos, con el objetivo de mejorar el rendimiento escolar, promover la justicia y la inclusión social e incidir en el desarrollo del país (p. 38).

Estos estudios de entidades nacionales e internacionales, han evidenciado múltiples factores en los procesos de aprendizaje y el desempeño existente en el sistema educativo costarricense. Ramírez y Quesada (2019) puntualizan que, dentro de estos factores, hay variables relevantes, como lo son: el estudiantado, sus familias, el personal docente, las metodologías para la enseñanza; las características sociales, económicas y culturales de los sitios donde se ubican los centros educativos y los procesos de gestión administrativa/curricular y de vinculación con la comunidad (p. 38).

Ante ello, el equipo de investigación de Administración Educativa de la UNED y el Colypro, desarrollaron una investigación que, según Ramírez y Quesada (2019), pretende formular indicadores de gestión administrativa, curricular y de vinculación con la comunidad, que den cuenta de buenas prácticas para el mejoramiento del desempeño escolar y puedan ofrecerse como insumo a personal directivo de centros educativos para el mejoramiento de su desempeño profesional, desde un enfoque de la calidad de la educación. Considerando dicho objetivo, el proceso ha contado con diversas etapas en las que se ha desarrollado una reflexión teórica y metodológica que permite construir un cuerpo de indicadores acorde con la demanda socioeducativa recién descrita. Por consiguiente, se ha especificado una serie de argumentos que han delimitado el espacio a investigar. De esta manera, se ha seleccionado la zona norte del país, por las condiciones diversas que esta posee; pues, por ejemplo, se pueden encontrar centros educativos unidocentes en zonas indígenas, diversidad de colegios (agropecuarios, técnicos y académicos), instituciones con modalidades para jóvenes y adultos, entre otras condiciones. En otras palabras, se trata de un escenario que cumple a cabalidad la posibilidad de entremezclar experiencias y construir, desde la diversidad de realidades sociales, una propuesta de gestión de la calidad.

De esta forma, el presente ensayo presenta una primera parte en la que se exponen los fundamentos teóricos que han sido considerados a nivel metodológico. En la segunda parte, se hace una revisión de la propuesta metodológica y, por último, se desarrolla una reflexión a partir de la ejecución del primer taller. Se concluye señalando algunos retos teórico-metodológicos. 


\section{Aproximación teórica desde el enfoque cualitativo}

Las características y fundamentos de la investigación cualitativa permiten una construcción entre el equipo investigador y el personal administrador de organizaciones educativas de la zona de estudio. A continuación, se detallan las principales características que reflejan estos fundamentos que han sido adoptados durante el proceso investigativo.

A nivel general, se puede iniciar con la característica que plantea Bernal (2010), citando a Bonilla y Rodríguez, quienes indican que el enfoque cualitativo es aquel que "se orienta a profundizar casos específicos y no generalizar, cualifica y describe el fenómeno según sea percibido dentro de la situación estudiada" (p. 60). En efecto, el enfoque cualitativo permite profundizar en los fenómenos de estudio y esta profundización permitiría la comprensión detallada de las realidades específicas.

Dorio, Sabariego y Massot (2009), ahondan en la caracterización de la investigación cualitativa dentro del libro "Metodología de la investigación cualitativa", coordinado por Bisquerra. Dichos autores delimitan la perspectiva hacia el ámbito educativo y toman la definición de Sandín, quien indica que:

La investigación cualitativa es una actividad sistemática orientada a la comprensión en profundidad de fenómenos educativos y sociales, a la transformación de prácticas y escenarios socioeducativos, a la toma de decisiones y también hacia el descubrimiento y desarrollo de un cuerpo organizado de conocimiento (p. 276).

Ahora bien, Dorio et. al. (2009), se basan en los argumentos de Eisner, desde los cuales plantean una serie de rasgos que, desde la investigación educativa, permiten la profundización de la información obtenida:

- Son estudios concentrados en contextos específicos, lo cual, según los autores, sugiere la reivindicación de la vida cotidiana como escenario básico con visión holística de los autores educativos (docentes, estudiantes, directores, entre otros).

- El grupo investigador participa dentro del proceso de construcción de conocimiento, rompiendo con el esquema del investigador fuera del objeto de estudio: el "yo" investigador, en un mismo espacio de los "yoes" participantes.

- Posee una naturaleza interpretativa por doble motivo: a) descubrir significados que van más allá de la información brindada, esto por medio de la observación de lo que hacen y el significado de lo que hacen los participantes del proyecto; b) analiza con profundización lo que pasa y analiza lo observado, indagando más allá de la información obtenida.

- El uso del lenguaje como factor importante: pretenden la reconstrucción de las realidades de las personas participantes, por medio del "dice y hace", permitiendo un mayor protagonismo y voz dentro de las situaciones estudiadas.

- Atención a lo concreto: se interesa en la profundización del grupo de estudio, lo cual, permite validar la singularidad de los acontecimientos (pp. 278-279).

En síntesis, el proyecto ha optado por un proceso de construcción de conocimiento que parte de las situaciones particulares de las personas participantes, ahondando en las situaciones, conocimientos y sus expresiones, con el fin de comprender a profundidad la perspectiva sobre la gestión y calidad de los indicadores desde el personal de administradores educativos. 


\section{Investigación acción}

El segundo elemento a describir dentro de la propuesta metodológica que ha sido construida para esta investigación, refiere a la perspectiva de la investigación acción (IA). En efecto, este tipo de investigación ha sido un pilar teórico-metodológico dentro del proceso que se ha desarrollado por el equipo de investigación. A continuación, se presentan sus características principales, así como su definición y aplicación en el ámbito educativo. Para ello, se tomarán los aportes de Colmenares y Piñero (2008), quienes delimitan fundamentos teóricos y metodológicos relevantes.

Para definir la IA, Yuni y Urbano, citados por Colmenares y Piñero (2008), la enmarcan como "un modelo de investigación de mayor compromiso con los cambios sociales", y esto implica el valor de la interpretación de las realidades en las que "Ios propios actores puedan comprometerse en procesos de cambio personal y organizacional" (p. 104). En efecto, esta definición de la IA implica una posición epistemológica y ontológica frente a las realidades sociales y, por tanto, la describen como algo más que un método:

Se asume una postura ontoepistémica del paradigma socio-crítico, que parte del enfoque dialéctico, dinámico, interactivo, complejo de una realidad que no está dada, sino que está en permanente deconstrucción, construcción y reconstrucción por los actores sociales, en donde el docente investigador es sujeto activo en y de su propia práctica indagadora (p. 104).

Ahora bien, la IA no solamente queda como una posición epistemológica y ontológica de la investigación, sino que Colmenares y Piñero (2008) la trasladan al ámbito educativo. Para ello, referencian a Restrepo Gómez, quien indica que la IA Educativa es un instrumento que puede permitir a los diversos actores educativos ser aprendices de la vida, de las formas enseñanza, de "cómo aprender a aprender, cómo comprender la estructura de su propia práctica y cómo transformar permanente y sistemáticamente su práctica pedagógica" (p. 105).

Por consiguiente, la IA educativa es una clara opción de corte metodológico, la cual permite las posibilidades de ampliación de conocimiento a partir de las perspectivas y reflexiones que van dando las personas involucradas dentro del proceso investigativo. De esta forma, tal y como lo manifiestan Colmenares y Piñero (2008), la IA Educativa es una forma clara de:

Explorar los actos educativos tal y como ocurre en los escenarios naturales dentro y fuera del aula (en la institución educativa en general); éstos pueden ser actos pedagógicos, administrativos, de gestión, de acción comunitaria, entre otros; se trata no solo de comprender una situación problemática en donde estén implicados los actores sociales educativos (docentes, estudiantes, representantes, entre otros), sino de implementar respuestas prácticas o acciones que permitan mejorar y modificar tal situación, y registrar y sistematizar toda la información posible que sobre el cambio se esté observando (p. 105-106).

En síntesis, la IA educativa permite una herramienta teórica y metodológica que pretende ser guía y alternativa, construyéndose y deconstruyéndose a partir de las diversas realidades socioeducativas, analizadas por parte de todas las instancias involucradas: grupo investigador - docentes - directores estudiantes, etc.

\section{Grupos focales}

Por último, en el marco de definición relacionado con la técnica de recolección de información, se contempló el grupo focal a partir de las características indicadas anteriormente, tanto con el enfoque cualitativo como en el método de la IA Educativa. 
Abarca, Alpízar, Sibaja y Rojas (2013), tomando a Frey y Patton, definen grupo focal como una entrevista grupal, aunque no todas las entrevistas grupales son grupos focales, puesto que es una técnica de investigación donde distintos participantes de un mismo contexto social pueden intercambiar criterios o información de manera simultánea (p. 160). Para los autores, el término focal "obedece a que la técnica se limita al estudio de un número reducido de temas, incluso quien modera debe mantener a los participantes circunscritos en los límites del conjunto de temas" (p. 161).

Asimismo, para la caracterización de la técnica de grupos focales, se tomó la propuesta por Morgan, citado en Balcázar, González-Arratia, Gurrola y Moysén (2013), que la describe de la siguiente forma:

- Tiene como propósito principal recopilar la información asociada a conocimientos, actitudes, sentimientos, creencias y experiencias que otras técnicas de investigación no permitirían.

- Es una modalidad de entrevista abierta y estructurada, que permite una conversación de grupo en el que el investigador guía algunas temáticas de interés para la investigación.

- Permite un intercambio de experiencias entre las personas que componen el grupo, por lo cual se comparten conocimientos sobre el tema y se retroalimentan entre sí.

- La interacción social es la característica fundamental de los grupos focales, ya que la dinámica creada entre los participantes permite resaltar las concepciones de las realidades, la posibilidad de interactuar entre sí por esas experiencias.

- Permite una aproximación multidimensional de las temáticas de interés, donde cada integrante investigador puede obtener información, tomar nota sobre el comportamiento grupal (tanto en términos de reacciones, actitudes y formas de comunicación no verbal).

- Permite obtener una descripción global de conocimientos, actitudes y comportamientos sociales de colectivos sociales y la priorización de la información obtenida por parte del equipo investigador (p. 126-127).

Por último, es importante mencionar que los diversos autores mencionan la relevancia de la persona moderadora dentro de la aplicación del grupo focal. En efecto, según Abarca et al. (2013), esta posee las siguientes características:

- Debe ser líder con diversas habilidades sociales para mantener la dirección de la actividad y motivar a las personas participantes a dar sus respuestas.

- Debe tener perfecto conocimiento de la guía de preguntas generadoras y decidir variar el orden a partir de las reacciones del grupo.

- Debe tener la plasticidad para ampliar información valiosa que se obtenga durante la discusión.

- Debe generar un ambiente de confianza, detectando los lenguajes verbales y no verbales dentro del grupo, así como el tipo de relación que establecen entre sí (p. 173).

\section{Fases de ejecución}

Abarca et al. (2013) plantean una serie elementos para la ejecución de los grupos focales. Es a partir de estos puntos que se han ejecutado las fases dentro de la investigación realizada. Para ello, se hará una breve descripción que se profundizará en el siguiente apartado.

- Especificación de las características de la población de interés: se seleccionó la zona de San Carlos, por tener características diversas similares a las compuestas a nivel nacional, es decir, unidocentes, 
indígenas, secundaria académica, secundaria técnica, secundaria agropecuaria, modalidades para jóvenes y adultos, preescolar, entre otros. Para ello, se hizo una muestra.

- Selección de los sujetos: se seleccionó personal administrador educativo, ya que son los encargados de analizar la gestión e indicadores de calidad, según el Ministerio de Educación Pública (MEP).

- Selección del espacio de reunión: se tomó en consideración la participación de la Regional de San Carlos del MEP, por lo cual se llevó a cabo dentro de las instalaciones de la Regional, punto de fácil ubicación para las personas.

- Convocatoria de las sesiones: se coordinó con la Dirección Regional de Educación (DRE) de San Carlos del MEP.

- Diseño del instrumento guía de entrevista: a partir de las dimensiones constituidas durante el proyecto, se construyeron las preguntas guías por dimensión.

- Obtención de datos mediante la discusión focalizada: se dividió en tres grupos, tomando en consideración las tres dimensiones. Cada grupo aportaba a las dimensiones. Cada grupo constaba de dos moderadores.

- Análisis de la información: se hizo grabación de las discusiones, así como un paleógrafo final. De ahí se obtendrá el análisis de la información, contrastada con apuntes principales.

\section{Construcción y definición de las dimensiones}

A continuación, se describe la construcción metodológica aplicada en el proceso, considerando la formulación y definición de las dimensiones de análisis, la conformación de las preguntas guías y los pasos realizados para la planificación y ejecución del grupo focal. La metodología para la construcción y definición de las dimensiones fue sustentada mediante la revisión extensa de material bibliográfico, lo que permitió una mejor comprensión del sistema y la realidad socioeducativa costarricense. A partir de esto, se logró sistematizar los principales indicadores de gestión educativa, por medio de las tres dimensiones propuestas en Ramírez y Quesada (2019): (a) la administrativa, (b) la curricular y (c) la de vinculación con la comunidad.

\section{Dimensión administrativa}

Tomando como base la propuesta dimensional por parte de Ramírez y Quesada (2019), en relación con la dimensión administrativa, se identificaron cuatro parámetros sobre los cuales se sustenta, a saber:

- Gestión del Centro Educativo: enfocado en aspectos de infraestructura y demás elementos físicos. Se fomenta la búsqueda de espacios adecuados para la población estudiantil, docente y administrativa, así como ambientes libres de contaminantes.

- Gestión del talento humano docente: se relaciona con gestiones que fortalezcan el quehacer docente, esto mediante la promoción de condiciones óptimas, el fortalecimiento de las motivaciones y la comprensión del contexto en que se desenvuelve cada estudiante, tal y como lo definen Ramírez y Quesada (2019).

- Gestión articuladora con la comunidad: este parámetro, basado en Ramírez y Quesada (2019), se enfoca en la implementación de acciones y estrategias desarrolladas para fomentar un mayor involucramiento de las familias y la comunidad con el centro educativo. 
- Gestión familiar y redes de apoyo: se refiere a la capacidad del centro educativo para articular a las familias en la toma de decisiones, así como en la construcción de acciones y estrategias para el mejoramiento de las condiciones educativas y el fortalecimiento de las capacidades académicas, curriculares y profesionales de la institución, tal y como lo manifiestan Ramírez y Quesada (2019).

\section{Dimensión curricular}

Esta segunda dimensión, según Ramírez y Quesada (2019), involucra tres parámetros, uno propiamente curricular y otros dos enfocados en los recursos y el contexto donde se desenvuelve el centro educativo.

- Curricular: la propuesta expuesta en Ramírez y Quesada (2019) contempla aspectos de planificación y evaluación institucional. En cuanto a la planificación, se refiere al cumplimiento de los programas educativos, conocimiento del plan de estudio, estrategias de programación, metas y objetivos institucionales, el desarrollo del Plan Anual de Trabajo y la formación de equipos. En cuanto a la evaluación se orienta a la supervisión y el seguimiento docente, la articulación del equipo curricular, prácticas evaluativas, el acompañamiento a la población estudiantil y el manejo de resultados.

- Material: se refiere a los recursos institucionales tales como espacios de estudio, bibliotecas, implementos tecnológicos en el proceso de aprendizaje y la organización de la infraestructura, según lo expuesto en Ramírez y Quesada (2019).

- Cultura: este parámetro, según lo planteado por Ramírez y Quesada (2019), toma en consideración el ambiente en términos de estructura curricular: principios institucionales; procedimientos para el personal administrativo, docente y estudiantil; participación en los espacios del centro educativo; medios de comunicación y reunión de los actores educativos; prestación de los servicios; liderazgo del director y seguimiento de las acciones docentes. Además, según los autores, contempla la formación del recurso humano mediante capacitaciones, equipos de formación continua y el desarrollo de competencias profesionales. Finalmente, el currículo intercultural comprende e incluye a la población migrante.

\section{Vinculación comunitaria}

Por último, la dimensión comunitaria, según Ramírez y Quesada (2019), se basa en la relación que posee el centro educativo con respecto a la comunidad en la que se encuentra. Este se define de acuerdo a dos parámetros planteados:

- Acciones educativas comunitarias: su objetivo, según el planteamiento de Ramírez y Quesada (2019), es generar espacios de comunicación e intercambio para mejorar las condiciones educativas del estudiantado y construir una retroalimentación cultural mutua (p. 43).

- Acciones referidas a la inclusión escolar: para Ramírez y Quesada (2019), estas acciones implican proyectos educativos entre la institución y las organizaciones de la comunidad como mecanismos de inclusión, posibilita la pronta visualización de estudiantes en situación de vulnerabilidad educativa y aquellos que se encuentran por fuera del sistema educativo (p. 43).

A partir de estas dimensiones y sus respectivos parámetros, se formulan distintas preguntas y subpreguntas o temas de interés que permitieron dirigir la discusión. El siguiente apartado gira en torno a estas formulaciones. 


\section{Construcción del instrumento: guía de preguntas}

Las preguntas que se formulan para incentivar y dirigir la discusión en un grupo focal son fundamentales para satisfacer el propósito de la convocatoria (Abarca, et al. 2013).

Por lo tanto, se parte de preguntas generales hasta llegar a las más específicas, ordenadas según prioridad en cada dimensión. A continuación, se establecen las preguntas generadas por el equipo investigador para recabar la información.

\section{Dimensión administrativa}

- ¿Cuentan los(as) directores(as) de los centros educativos con indicadores de gestión que les permitan dar seguimiento y tomar decisiones de índole administrativa, institucional y comunitaria? En este aspecto se contempla la evaluación del espacio físico (el deterioro y estado de las instalaciones de la institución). Además, se indaga por el plan de mejora de infraestructura, de acuerdo a lo estipulado por la Ley No. 7600.

- ¿Cuáles instrumentos posee el centro educativo para realizar la evaluación de desempeño de estudiantes, docentes, personal administrativo y personal de mantenimiento? Consultar acerca de los elementos que incorpora el perfil del o la docente, así como los mecanismos de sistematización y seguimiento para la evaluación de desempeño del personal.

- ¿Participa la comunidad de algunas propuestas que se realicen para mejorar el desempeño escolar de los y las estudiantes? En este apartado se indaga sobre aquellos espacios abiertos que permitan el acercamiento de la comunidad a la institución.

- ¿Participa la comunidad en la toma de decisiones y la construcción de alternativas para fortalecer la capacidad y alcance del centro educativo? Considerar la participación de padres y madres de familia, así como miembros de la comunidad en el Comité de Apoyo de la institución. Así mismo, el registro de actividades, cuyo fin sea la proyección hacia la comunidad.

\section{Dimensión curricular}

- ¿Qué aspectos de la planificación curricular requieren mayor abordaje para garantizar la calidad en la gestión institucional? ¿Cuentan los directores y las directoras con herramientas que permiten una clara y eficiente implementación de los elementos contemplados en la planeación curricular institucional? Se busca recabar información sobre acciones que fomenten la mejora continua de las prácticas evaluativas.

- ¿Los recursos institucionales están direccionados de forma eficiente, buscando su máximo aprovechamiento? Indagar si promueven el uso de los diversos espacios institucionales, cuentan con mecanismos de seguimiento para registrar su uso; además, se promueve el uso de la tecnología en los procesos de aprendizaje y se realiza un uso eficiente de la infraestructura disponible.

- ¿ ¿De qué manera la estructura organizacional favorece al centro educativo y sus propósitos? ¿Qué acciones se realizan para la promoción y el fortalecimiento de la cultura organizacional? ¿El personal docente participa de forma activa en los procesos de formación continua para el fortalecimiento de las competencias profesionales? ¿Cómo se detectan las necesidades de formación continua en el personal docente del centro educativo? ¿Qué mecanismos existen para estimular la participación 
de profesores en los procesos de formación continua? ¿Se brinda seguimiento o acompañamiento para la aplicación de los conocimientos adquiridos en el aula?

- Con respecto al currículo intercultural, se indaga sobre la integración de la población migrante al centro educativo. ¿Existen estudios periódicos que caracterizan a la población migrante estudiantil en el centro educativo? Se toman en cuenta acciones que se desarrollan o podrían desarrollarse a nivel interno para fortalecer el currículo intercultural e involucrar a la población migrante.

\section{Vinculación comunitaria}

- ¿Existen espacios periódicos que inviten al trabajo conjunto entre la comunidad y el cuerpo docente y administrativo del centro educativo, para el análisis de las situaciones socio-educativas locales? ¿Cómo se han conformado estos espacios o, en su defecto, por qué no se han conformado? ¿Cuáles grupos organizados de la población comunitaria han sido parte o podrían serlo? ¿Cuentan con una estrategia de seguimiento para mantener estos espacios?

- ¿Qué función cumplen los padres y madres de familia y otros agentes sociales de la comunidad, en la mejora del desempeño escolar de los y las estudiantes? ¿Existe participación directa o parcial por parte de padres y madres de familia en propuestas pedagógicas y/o evaluativas? ¿Qué mecanismos de seguimiento se dan a estas propuestas?

- ¿El centro educativo realiza diagnósticos sobre los temas de identidad comunitaria con el fin de ser incorporadas dentro del proceso de formación de la población estudiantil? ¿Qué aspectos contempla dicho diagnóstico? ¿Qué tanto inciden los resultados el diagnóstico en la planificación de año lectivo y de posibles proyectos?

- ¿El centro educativo ha conformado proyectos socioeducativos vinculados con la comunidad a partir de ideas construidas en conjunto? ¿Qué resultados han obtenido? ¿Cuáles han sido las problemáticas a solventar? ¿Qué posibles acciones se podrían programar para formular proyectos en conjunto con la comunidad?

Estos bloques de preguntas formuladas por el equipo investigador constituyeron una guía que permitió delimitar los temas a tratar y permitió a las personas moderadoras hacer una distribución eficiente del tiempo durante la discusión. Seguidamente, se establecen los pasos metodológicos realizados para la planificación y ejecución del grupo focal.

\section{Planificación y ejecución del grupo focal}

La planificación de los grupos focales generó una serie de trámites administrativos y logísticos necesarios para el éxito de su implementación. Un aspecto fundamental fue la adecuada coordinación con autoridades del Ministerio de Educación Pública para garantizar la convocatoria y participación de las personas encargadas de la administración educativa.

La muestra de centros educativos consultados fue seleccionada por el equipo investigador. La misma fue conformada por 7 colegios y 27 escuelas pertenecientes a la DRE de San Carlos, Circuitos Dos, Tres y Cuatro.

El proceso implicó la convocatoria y reunión de 34 administradores educativos, así como 4 asesores regionales que conforman el Equipo Regional de la Calidad en la DRE de San Carlos. 
En cuanto a la ejecución del grupo focal, se inició con una introducción sobre la política educativa costarricense y sus implicaciones en la administración educativa, realizada por uno de los miembros del equipo investigador. Seguidamente, se procedió a dividir a los participantes en tres subgrupos, cada uno dedicado a una dimensión específica, con un tiempo de 30 minutos para discutir sobre las preguntas y temas propuestos. Al cabo de los 30 minutos, se rotaban las dimensiones a discutir para abarcar todos los temas con los diferentes grupos y así recolectar la información de manera completa.

Durante la tarde, otro miembro del equipo investigador generó un conversatorio sobre la Agenda 2030 para el Desarrollo Sostenible y su incidencia en la administración del sistema educativo. Al cierre de la jornada de trabajo, se instó a las personas participantes a realizar un papelógrafo con aquellos retos que deben enfrentar desde su labor, para satisfacer las demandas educativas y cumplir con los estándares de la calidad en sus centros educativos.

\section{REFLEXIONES EN TORNO AL TEMA}

\section{Reflexiones iniciales dentro del marco de investigación}

Documentos tales como el "Centro Educativo de la Calidad como Eje de la Educación Costarricense" (CSE, 2008), la nueva política educativa "La persona: centro del proceso educativo y sujeto transformador de la sociedad" (CSE, 2017), así como la propuesta del Modelo de Evaluación de la Calidad de la Educación Costarricense (MECEC, 2013), pretenden ser el marco regulador para empoderar el rol del director y permitir que este se concentre en el fortalecimiento de la calidad educativa. No obstante, esto no se observó en la discusión con las personas administradoras, dada la dificultad que manifiestan para hacer seguimiento y evaluar las metas y objetivos de la institución, así como para detectar y canalizar las demandas de la comunidad educativa.

Adicionalmente, los participantes describieron un reconocimiento al MECEC como herramienta de planificación educativa que depende de un trabajo colaborativo y no solo de la persona directora como profesional responsable. Por lo tanto, es necesaria la concientización por parte de los diferentes actores relacionados con la gestión educativa, entre ellos, la dirección de la institución, los supervisores y la DRE, de la necesidad de coordinar acciones que se orienten los procesos de mejoramiento hacia las dimensiones de gestión curricular y comunitaria, mientras que, para la dimensión administrativa, sea menos entrega de información y control.

\section{Líneas de análisis a partir de la ejecución del grupo focal}

El objetivo de este ensayo es reconocer y explorar aspectos metodológicos del uso del grupo focal en el contexto de la investigación de gestión e indicadores de calidad con administradores educativos. El proceso brindó una oportunidad para explorar ventajas y desventajas de los grupos focales cuando se analizaron aspectos metodológicos, así como el contexto de la recopilación de datos, la población participante, y, por último, el papel de la persona moderadora. A continuación, se presentan elementos propios que inciden en la aplicación de un grupo focal. Se detalla el cómo cada uno fue considerado y tratado durante la aplicación de la técnica grupo focal.

\section{El lugar}

Como lo exponen diferentes autores (Amezcua, 2003; Abarca et. al., 2013; Martínez, 2015), la decisión de escoger el lugar para realizar el grupo focal es un elemento importante y se complementa con la 
selección de los participantes, respecto del lugar se siguieron las recomendaciones para realizar a saber: (a) comodidad, (b) neutralidad, (c) ruido. Por lo tanto, del lugar elegido se puede decir que estuvo acorde debido a que propició en los participantes una actitud de participar activamente de las preguntas generadoras. Además, conforme a estos postulados teóricos la discusión en cada subgrupo osciló entre 30 y 45 minutos, de manera que todo el proceso del grupo focal tuvo una duración aproximada de dos horas.

\section{Ética}

Otro aspecto importante tal como lo plantean (Campos, 2015; Campos \& Madriz, 2015) fueron las cuestiones éticas para los procesos investigativos de manera tal que a los directores se les invitó a que participaran de manera voluntaria en los grupos focales. Al inicio de la sesión, se tuvo un espacio para informar sobre los objetivos de la investigación y su condición de informantes en la actividad a realizar. Se solicitó de forma previa a los directores su autorización para grabar la sesión y, además, se garantizó confidencialidad de la información obtenida en los grupos focales.

\section{Moderadores}

El papel de moderador es importante en la conducción de los grupos focales, según lo manifiesta Krueger y Casey, citados en Onwuegbuzie, Dickinson, Leeche y Zoran (2011). En efecto, para los autores, en este tipo de espacios se genera información valiosa $y$, por tanto, el efecto que pueda construir el moderador frente a las personas informantes es clave. Aspectos como la edad podrían influir, especialmente en el caso de investigadores más jóvenes que presentan gran diferencia de edades respecto del grupo. A modo de ejemplo, durante el trabajo en subgrupos, cada moderador debería crear un clima propicio donde cada participante pudiera expresarse libremente, además de considerar reglas básicas durante su desarrollo, como mantener el hilo conductor de la temática y conseguir ser concretos ante las preguntas. Por lo tanto, en un momento que se quería mantener el foco de la temática, un participante manifestó cierta reticencia a la confrontación de opiniones, bajo el supuesto de que pudiesen interpretarse como crítica a su propia actuación profesional. Incluso, cabe destacar que al final de la sesión expresó inquietudes sobre el hecho de que se estuviera realizando una confrontación de sus acciones, porque él tiene un puesto con autoridad formal.

\section{Interacción en los grupos}

Una de las ventajas de los grupos focales son los procesos de interacción, de manera tal que los participantes pueden reaccionar y construir sobre otras respuestas. Esto ocurrió con frecuencia en los tres subgrupos: los participantes a menudo recordaron ejemplos de sus experiencias propias en el momento que otro participante contaba lo vivido; por lo tanto, se puede afirmar que tuvieron la posibilidad de expresar en forma abierta sus opiniones acerca de la gestión pedagógica y que su relación con los investigadores se dio en un clima de confianza.

Sin embargo, puede ser más difícil moderar un grupo focal con un número de participantes amplio y que ya se conozcan (como fue el caso de esta experiencia), porque existe una mayor probabilidad de dinámicas grupales más complicadas, particularmente si algunos miembros tienden a dominar el uso de la palabra e inhiben a otros participantes. 


\section{Líneas iniciales de análisis desde el enfoque de Administración Educativa}

Para la DRE de San Carlos, es una necesidad en materia de gestión lograr en el nivel micro consolidar la propuesta para el proceso de fortalecimiento de la gestión de calidad, tal como se plantea en la nueva política "La persona: centro del proceso educativo y sujeto transformador de la sociedad".

...transparencia en los procesos de gestión regionales y se optimizará el tiempo de personal docente y administrativo, de forma que puedan concentrarse los esfuerzos en el fortalecimiento de la calidad educativa... (CSE, 2017, p. 23).

También, se evidencia que la DRE de San Carlos cuenta con un marco regulador para el logro de una gestión de calidad, además, cuentan con algún grado de autonomía, estructura y los mecanismos para la ejecución del proceso de mejoramiento de la calidad. No obstante, la capacidad de los centros educativos de ser autónomos y realizar los ajustes para enfrentar sus distintas realidades aún es débil, debido principalmente a que es prioritario todo lo que demanda el nivel central del MEP.

Tal como lo manifiestan Elizondo (2005) y Aguerrondo (2007), las organizaciones educativas deben ser permeables a los cambios del entorno y propiciar procesos de identidad y autonomía que les permitan hacer frente a los múltiples problemas educativos, de manera que los administradores de la educación orienten su labor en la dimensión pedagógica e impulsen procesos de mejora y una cultura permanente de autoevaluación que permita una toma de decisiones relevante y contextualizada para la organización educativa.

\section{REFLEXIONES FINALES}

\section{Retos teóricos}

Abordar la construcción de los instrumentos de recolección de información desde el marco referencial de la disciplina, permitió realizar una mirada a las transformaciones que, desde lo teórico, ha tenido la administración educativa y enfocarse, durante el grupo focal, hacia aspectos esenciales para el director como actor fundamental de un entorno social en proceso de cambio educativo.

El aporte desde el enfoque cualitativo a la comprensión de problemas de calidad en la educación, entre los que se encuentra la construcción de indicadores, resultó significativo porque exige un abordaje con diferentes miradas. El conocimiento sobre indicadores que posee la población de las personas directoras varía mucho entre sí, por lo cual, hablar de la calidad y de cómo medirla, implica una unificación de criterios que permitan un abordaje más acertado. Esto, por tanto, genera un reto enorme para el sistema educativo, quien debería profundizar en las referencias utilizadas para construir conocimiento que no se aleje de las realidades sociales de los centros educativos.

\section{Retos metodológicos: planificación y ejecución}

El grupo focal, como técnica de recolección de información, requiere del investigador educativo un riguroso proceso de planificación para alcanzar los objetivos de investigación. Esto se debe, en primera instancia, a que no ha sido una tendencia en el área de la administración educativa y, en segunda, a la 
dinámica de trabajo de los gestores educativos, la cual consiste en ejecutar acciones en grupos, tanto de profesionales de la educación, padres de familia y la comunidad.

En este tipo de procesos, el tiempo suele ser uno de los principales retos. De acuerdo al número de participantes y la cantidad de minutos disponibles para dedicar a cada dimensión, se debe restringir según el número de preguntas a formular. Además, en grupos grandes, el tiempo de respuesta de cada participante también debe limitarse, esto para poder escuchar la visión de todos o al menos de la mayoría.

Por otro lado, la persona que modera o facilita el grupo focal requiere contar con una amplia gama de habilidades que le permitan sistematizar los diferentes aportes de las personas participantes, así como dirigir y delimitar la discusión en torno a los puntos de interés. Además, una habilidad fundamental para desempeñarse en este tipo de técnicas es la capacidad para no inducir o direccionar una respuesta anticipada, sesgando la información recolectada; en contraposición se deben fomentar las respuestas reflexivas, críticas, desde la opinión de cada participante.

El grupo focal, como método de recolección de información cualitativa, generó mucha riqueza de información; además, plantea la necesidad de construir procesos de mejoramiento de la calidad con espacios de análisis más profundos, debido a que los contextos socio-educativos son cada vez más complejos, producto de las diferentes situaciones que rodean la educación y su calidad.

\section{Retos desde la Administración Educativa}

Es importante reconocer el esfuerzo realizado desde el MECEC en procura de una cultura de calidad en las instituciones educativas. Esto permite contar con un conjunto de estándares, criterios, procesos y productos para desarrollar una gestión de calidad. No obstante, los cambios en las políticas educativas generan un primer desafío para las personas administradoras de la educación, porque contiene un énfasis en la importancia de la gestión curricular, la administración orientada a resultados y la vinculación de la educación al contexto social, en consecuencia, exige la construcción de indicadores en la dimensión curricular más centrados en la tarea pedagógica.

Otro reto importante pareciera ser la consolidación de procesos de gestión comunitaria en las instituciones educativas; porque, desde los lineamientos de política, se marca una ruta para la generación de indicadores que midan estos procesos tales como, comunidad educativa abierta, formación de redes regionales y construcción de alianzas.

Finalmente, es necesario gestionar procesos de capacitación que contribuyan con la comprensión de la comunidad educativa de los procesos de construcción de indicadores que les permita gestionar adecuadamente este momento de cambio.

\section{REFERENCIAS}

Abarca, A., Alpízar, F., Sibaja, G. \& Rojas, C. (2013). Técnicas cualitativas de investigación. San José, Costa Rica: Editorial de la Universidad de Costa Rica.

Aguerrondo, I. (2007). Racionalidades subyacentes en los modelos de planificación educativa. Revista Brasileira de Política e Administração da Educação, v. 23(3), 463-481.

Amezcua, M. (2003). La entrevista en grupo. Características, tipos y utilidades en investigación cualitativa. Enfermería clínica, 13(2), 112-117. 
Balcázar, P., González-Arratia, N., Gurrola, G. \& Moysén, A. (2013). Investigación cualitativa. Estado de México, México. Universidad Autónoma de Estado de México.

Bernal, C. (2010). Metodología de la Investigación administración, economía, humanidades y ciencias sociales. Bogotá, Colombia: Pearson Educación.

Campos, J. (2015). Cómo hacer un Trabajo Final de Graduación. Lineamientos para la Escuela Ciencias de la Educación. San José, Costa Rica: EUNED.

Campos, J. \& Madriz, L. (2015). Investigación-Acción en Contextos Educativos. San José, Costa Rica: EUNED.

Colmenares, A. \& Piñero, M. (2008). La investigación acción. Una herramienta metodológica heurística para la comprensión de realidades y prácticas socio-educativas. Revista Laurus, 14(27), 96-114. Recuperado de http://www.redalyc.org/pdf/761/76111892006.pdf

Consejo Superior de Educación. (2008). El Centro Educativo de Calidad como Eje de la Educación Costarricense. San José, Costa Rica.

Consejo Superior de Educación. (2017). La persona: centro del proceso educativo y sujeto transformador de la sociedad. San José, Costa Rica.

Dorio, I., Sabariego, M. \& Massot, I. (2009). Características generales de la metodología cualitativa. En Bisquerra, R. (Coordinador), Metodología de la investigación educativa (pp.275-292). Madrid, España: Editorial La Muralla.

Elizondo, A. (coord.) (2005). La nueva escuela. Dirección, liderazgo y gestión escolar (t. I). Distrito Federal, México: Paidós.

Martínez, N. (2015). Reseña metodológica sobre los grupos focales. Diálogos 9, 47-53.

Onwuegbuzie, A., Dickinson, W., Leech, N. \& Zoran, A. (2011) Un marco cualitativo para la recolección y análisis de datos en la investigación basada en grupos focales. Revista Paradigmas, 3(1), 127-157. Recuperado de https://dialnet.unirioja.es/descarga/articulo/3798215.pdf

Ministerio de Educación Pública. (2013). Modelo de Evaluación de la Calidad de la Educación (MECEC). San José, Costa Rica: Dirección y Gestión de la Evaluación de la Calidad. Departamento de Evaluación de la Calidad.

Ramírez, M. \& Quesada, J. (2019). Repensando los indicadores educativos: la gestión educativa, curricular y de vinculación con la comunidad. Revista Innovaciones Educativas, 21(30), 37-47. Recuperado de https://revistas.uned.ac.cr/index.php/innovaciones/article/view/2483/3153 